\title{
Evolución de los niveles de burnout en un estudio comparativo en cuidadores profesionales tras una situación de estrés postraumático
}

\author{
Juan P. Martínez ${ }^{1}$, Inmaculada Méndez², Esther \\ Secanilla ${ }^{3}$ y Elena González ${ }^{2}$ \\ ${ }^{1}$ Equipo de Orientación Educativa y Psicopedagógica del Altiplano (Murcia); \\ ${ }^{2}$ Universidad de Murcia; ${ }^{3}$ Universidad Autónoma de Barcelona
}

Tras un estudio previo realizado en cuidadores profesionales de personas con demencia y otras enfermedades en centros residenciales en el que se comparó dos muestras en distintas comunidades autónomas, Murcia y Barcelona. Partiendo de los resultados obtenidos en los cuales el $13.3 \%$ de la muestra global presentaba un agotamiento emocional alto, un $54.8 \%$ un nivel de despersonalización alto y un $10 \%$ una baja realización personal, se procede a realizar un análisis de los niveles actuales en las tres dimensiones para estudiar su evolución. Del mismo modo, se evalúa si en la población de Lorca (Murcia) dichos niveles han podido verse afectados por el terremoto acaecido el 11 de mayo de 2011, para lo cual se ha llevado a cabo una evaluación de posibles síntomas de un Trastorno por Estrés Postraumático (TEP). Se ha procedido a la administración de las mismas pruebas de la última investigación (escala Maslach Burnout Inventory -MBI- y Encuesta elaborada ad hoc para Cuidadores Profesionales) junto a la escala SA-45 forma abreviada de la SCL-90 y la Escala de Gravedad de Síntomas del Trastorno de Estrés Postraumático. Los resultados obtenidos reflejan diferencias entre ambas poblaciones en diversas variables analizadas y arrojan luz acerca de las consecuencias y el impacto de una situación traumática en la población.

Palabras clave: Burnout, estrés postraumático, cuidadores, demencias y estudio comparativo.

Changes in levels of burnout in a comparative study in professional caregivers after a situation of post-traumatic stress. Following a previous study on professional caregivers of people with dementia and other diseases in residential, was decided to compare two residential centers of two different regions, Murcia and Barcelona. Based on the results of which $13.3 \%$ of the global sample had a high emotional exhaustion, $54.8 \%$ a high level of depersonalization and $10 \%$ a reduced sense of personal accomplishment, we proceed to an analysis of current levels in the three dimensions to study their evolution. Similarly, we assess whether in the town of Lorca (Murcia), these levels have been affected by the earthquake on May 11, 2011, for which it has conducted an evaluation of possible symptoms of PostTraumatic Stress Disorder (PTSD). We proceeded to the administration of the same tests of the latest research (Scale Maslach Burnout Inventory -MBI- and an ad hoc survey conducted for professional caregivers) and the Scale SA-45 which is the short form from the original SCL-90, and the Severity of Symptoms Scale of Posttraumatic Stress Disorder. The results show differences between the two populations in different variables and they shed light on the consequences and impact of a traumatic event in the sample.

Key words: Burnout, post-traumatic stress, caregivers, dementia and comparative study.

Correspondencia: Juan Pedro Martínez Ramón. Equipo de Orientación Educativa y Psicopedagógica del Altiplano. Avenida de los Reyes Católicos, 34. C.P. 30520. Jumilla-Murcia (España). E-mail: juanpedro.martinez@murciaeduca.es 
El síndrome de "burnout" o síndrome de estar quemado en el trabajo es un factor común al espectro psicopatológico de las profesiones y oficios caracterizados por la interacción humana. El estudio de este concepto se inició con síntomas reportados por personal del sector servicios que se sentían cansados emocionalmente y que desarrollaban pautas de alejamiento hacia sus clientes y/o pacientes (Freudenberger, 1974). En concreto, está ampliamente aceptado que el síndrome de burnout está compuesto por tres dimensiones: agotamiento emocional, despersonalización y baja realización personal. Aquellas profesiones donde es necesario tener un contacto directo y continuo con otras personas tienen un mayor riesgo de sufrir este síndrome (Martínez, 2011).

Los cuidadores profesionales de personas mayores institucionalizadas con demencias y otras enfermedades son un colectivo diana por la naturaleza intrínseca de sus funciones de apoyo e interacción social habiéndose altos niveles en las dimensiones de burnout. Éstos sufren sobre todo las consecuencias del deterioro progresivo de la persona con Enfermedad de Alzheimer u otras demencias. Esta afirmación está abalada por una investigación previa que se realizó en distintas ubicaciones geográficas (Murcia y Barcelona). En ella se halló que el 13,3\% presentaba agotamiento emocional alto, un 54,8\% altos niveles de despersonalización y un $10 \%$ con baja realización personal (Méndez, Secanilla, Martínez y Navarro, 2011).

El personal de estas residencias consiguen aliviar las cargas familiares puesto que realiza unas funciones básicas con el afectado mientras los demás miembros de su familia pueden tratar de hacer otras actividades que no estén directamente relacionadas con su cuidado (Guilló, 2002). Obviamente, a los niveles de estrés de los cuidadores provocados por la persona dependiente, se suman otros como son las características personales (variables sociodemográficas), características del trabajo, ausencia de mecanismos como el apoyo social y las características específicas del puesto en interacción con las personas (Ortega y López, 2004).

En términos sociológicos, la pirámide poblacional se está invirtiendo de forma que cada vez es más la población que se aglutina en la parte superior de dicha pirámide mientras que es menor el porcentaje de población joven en edad de trabajar que puede mantener al otro sector (Pérez, 2010).

Además, existe una relación directa entre edad cronológica y la prevalencia de trastornos mentales orgánicos (Gázquez, Pérez, Lucas y Yuste, 2008). Todos estos datos justifican la necesidad de ondear más en la problemática del burnout en cuidadores profesionales.

Con respecto al trastorno por estrés postraumático, éste aparece en aquellas situaciones en las que una persona ha pasado por un episodio de gran carga emotiva y acompañado de altos niveles de estrés. Tradicionalmente fue estudiado en soldados que volvían del campo de batalla con síntomas similares: mucho estrés, miedo, sensación de estar reviviendo el suceso traumático en determinados momentos, etc. Los cuidadores profesionales de Lorca vivieron esta situación tras el terremoto acaecido el día 11 de mayo del 2011, con gran angustia y supuso un acontecimiento de gran conmoción para toda la población. De acuerdo con Bowman y Yehuda (2004), los sucesos adversos repercuten en el desarrollo de los trastornos emocionales. Para poder ser diagnosticado de TEP, el sujeto debe haber pasado por una situación traumática. Tras este suceso, desarrolla una serie de síntomas que se agrupan en tres bloques o categorías: reexperimentación del acontecimiento traumático, evitación persistente hacia los estímulos asociados con el trauma y 
aumento de la activación autonómica traducido en dificultades para conciliar el sueño o mantener el sueño, entre otras características.

Partiendo del estudio piloto anterior, la presente investigación tiene un doble objetivo:

1. Realizar un análisis de los niveles actuales en las tres dimensiones de burnout en la muestra murciana y barcelonesa para estudiar su evolución.

2. Evaluar si en la población de Lorca (Murcia), los niveles hallados se han podido ver afectados por el terremoto del 11 de mayo de 2011. Para registrar este último aspecto, se ha procedido a incluir dos escalas para la evaluación de síntomas del Trastorno por Estrés Postraumático (TEP).

Por tanto, se trata de un estudio longitudinal donde se va a estudiar el avance o retroceso de los síntomas descritos el año pasado en ambas residencias, analizando las diferencias significativas que puedan existir, y por otro lado, un estudio experimental pre-postest donde se va a estudiar la influencia del terremoto de Lorca en los cuidadores profesionales antes y después del suceso.

\section{METODO}

\section{Participantes}

La muestra de la investigación actual está compuesta por 19 cuidadores profesionales ( $N=10$ en Barcelona y $N=9$ en Murcia). El 89,5\% (17 sujetos) de la muestra total está compuesta por mujeres, mientras que el 10,5\% (2 sujetos) por varones. Si segmentamos la muestra en función de su centro laboral de procedencia, los dos hombres se ubican en Lorca $(22,2 \%)$ de forma que el $78,8 \%$ restante son mujeres. En Barcelona, el $100 \%$ está formado por mujeres. La franja de edad es amplia y oscila entre los 22 y los 62 años. La media es de 39,84 años $(D T=12,52)$.

\section{Instrumentos}

Se ha procedido a la administración de las mismas pruebas de la última investigación, es decir, el Maslach Burnout Inventory -MBI- (Maslach y Jackson, 1986) y la Encuesta elaborada ad hoc para Cuidadores Profesionales (Méndez, Secanilla, Martínez y Navarro, 2011) junto con la Escala de Gravedad de Síntomas del Trastorno de Estrés Postraumático (Echeburúa, Corral, Amor, Zubizarreta y Sarasua, 1997) y el Symptom Assessment-45 Questionnaire (SA-45) (Sandín, Valiente, Chorot, Santed y Lostao, 2008). Estas dos últimas sólo se han aplicado en la población murciana.

En primer lugar, el MBI es una herramienta reconocida por la comunidad científica para la valoración de las tres dimensiones del síndrome de burnout a través de 22 ítems. La dimensión "agotamiento emocional" (AE) está formada por los ítems 1, 2, 3, 6, 8, 13, 16 y 20. La "despersonalización” (DP) está compuesta por los ítems 5, 10, 11, 15 y 22. Y, por último, la "realización personal en el trabajo" (RP) queda definida por los ítems $4,7,9,12,17,18$ y 21. Se han utilizado los puntos de corte usados en el estudio de Seisdedos (1997) utilizando su estudio como población normativa (Tabla 1). 
De este modo, obtenemos los percentiles 33 y 66 dividiendo cada dimensión en tres segmentos o niveles: bajo, medio y alto. Aparecerá el cuadro sintomatológico completo del síndrome cuando aparezcan altos niveles de agotamiento emocional y despersonalización, y bajos niveles de realización personal en el trabajo (Olivares, Vera y Juárez, 2009). La consistencia interna de Alfa de Cronbach de las tres escalas fluctúa entre los valores 0,71 y 0,90 (Alarcón, Vaz y Guisado, 2002). La fiabilidad en test-retest en aproximadamente un mes oscila de 0,60 y 0,80 (Jackson, Shwab y Schuler, 1986).

Tabla 1. Puntos de corte en las dimensiones de burnout del MBI (Seisdedos, 1997)

\begin{tabular}{cccl}
\hline & Bajo & Medio & \multicolumn{1}{c}{ Alto } \\
\hline AE & menos de 18 puntos & de 19 a 26 puntos & más de 27 puntos \\
\hline DP & menos de 5 puntos & de 6 a 9 puntos & más de 10 puntos \\
\hline RP & menos de 33 puntos & de 34 a 39 puntos & más de 40 puntos \\
\hline
\end{tabular}

En segundo lugar, se ha utilizado un instrumento elaborado para la última investigación denominado "Encuesta a trabajadores de residencias de personas mayores" compuesta por 48 ítems distribuidos en cuatro bloques: características sociodemográficas; consumo de drogas; problemas a nivel laboral o familiar y hábitos deportivos y salud. Esta escala posee un índice de fiabilidad de Cronbach de 0,639 para el total de la muestra.

En tercer lugar, en la muestra de Lorca (Murcia) se ha llevado a cabo una evaluación de posibles síntomas de un Trastorno por Estrés Postraumático (TEP) utilizando la Escala de Gravedad de Síntomas del Trastorno de Estrés Postraumático. Se trata de una entrevista estructurada, heteroaplicada que permite evaluar los síntomas del trastorno de estrés postraumático así como su intensidad, según los criterios diagnósticos del DSM-IV (APA, 1994). Dicha escala consta de 17 ítems en formato tipo Likert de 0 a 3 según la frecuencia e intensidad de los síntomas (nada, una vez por semana o menos/poco, de 2 a 4 veces por semana/bastante, 5 o más veces por semana/mucho). Las escalas que forman dicho instrumento son: síntomas de reexperimentación (5 ítems), evitación (7 ítems) e hiperactivación (5 ítems). La puntuación resultante oscila en un rango de 0 a 51 en la escala global siendo 15 el punto de corte; de 0 a 15 en la subescala de reexperimentación siendo 5 el punto de corte; de 0 a 21 en la subescala de evitación con punto de corte de 6 y de 0 a 15 en la subescala de activación con un punto de corte de 4 . El coeficiente de fiabilidad test-retest en muestra clínica $(N=175)$ y en sujetos normativo $(N=463)$ es de $0,89(p=0,001)$ con un intervalo de 4 semanas. El índice de consistencia interna a través del coeficiente alfa de Cronbach para el conjunto de la muestra fue de 0,92 (Echeburúa et al., 1997).

Finalmente, junto al instrumento anterior, se ha utilizado el Symptom Assessment-45 Questionnaire (SA-45) (Sandín et al., 2008). Se trata de un instrumento de autoinforme de síntomas psicopatológicos derivado de las dimensiones que evalúa el 
SCL-90. Consta de 45 ítems en una escala tipo Likert (nada/un poco presente, moderadamente, bastante y mucho o extremadamente) que se dividen en nueve escalas de 5 ítems cada uno, de las que en nuestro estudio se han seleccionado las siguientes: depresión, somatización, ansiedad, sensaciones interpersonales. En cuanto a la fiabilidad de la prueba, el coeficientes alfa de Cronbach para el total de la prueba fue de 0,95, siendo para cada una de las escalas: depresión $(\alpha=0,85)$; somatización $(\alpha=0,80)$; ansiedad $(\alpha=0,84)$ y sensaciones interpersonales $(\alpha=0,84)$. Alvarado, Sandín, ValdezMedina, González-Arratia y Rivera (2012) hacen especial hincapié en que este instrumento presenta una constatada validez transcultural.

\section{Procedimiento}

Puesto que esta investigación parte de un estudio previo, inicialmente se realizó un estudio piloto en el que se seleccionaron dos centros residenciales de personas mayores en Lorca (Murcia) y Barcelona, llevándose a cabo una entrevista con los Directivos de dichos centros para describir los objetivos de la investigación y aclarar los instrumentos de evaluación. Una vez obtenido su aprobación, se comenzó a recopilar datos. La muestra fue seleccionada aleatoriamente entre el personal de ambos centros informando a los cuidadores profesionales de cuáles eran los objetivos del estudio, cómo se cumplimentaban los cuestionarios y acerca de la confidencialidad de los resultados. Los cuestionarios que no estaban correctamente cumplimentados se eliminaron y se produjo mortandad experimental en algunos sujetos. Los resultados obtenidos suponen el pretest de nuestra investigación actual y fueron codificados en una base de datos del programa SPSS (versión 15.0). A la hora de realizar el postest, se seleccionaron a los mismos sujetos que participaron en el estudio piloto y se les pasó la misma batería de pruebas tanto a la residencia de Murcia como de Barcelona. Además, los cuidadores profesionales de Lorca (Murcia), cumplimentaron dos cuestionarios específicos para evaluar el Trastorno por Estrés Postraumático, teniendo como puntuaciones de referencia las obtenidas en el pretest. Se han utilizado técnicas estadísticas descriptivas e inferenciales como el análisis de frecuencias y las tablas de contingencia (prueba Chi-Cuadrado) y el coeficiente de correlación para hallar significatividad.

\section{RESULTADOS}

Se ha realizado un análisis descriptivo de la muestra centrándose en las características más relevantes. Con respecto al estado civil, el 57,9\% está casado, el $36,8 \%$ está soltero y el 5,3\% viudo. Con relación al nivel de estudios, el 36,8\% presenta estudios universitarios, el $26,3 \%$ bachillerato y el resto estudios medios o primarios. Tan solo el 5,3\% no tiene estudios primarios concluidos. El entorno familiar ha sido otro de los campos estudiados. Así, observamos que el 57,9\% de los cuidadores encuestados no 
tiene hijos, seguido de un $21,1 \%$ con dos hijos. El 68,4\% vive con su pareja, el 5,3\% convive con su padre y el $15,8 \%$ con su madre. A la hora de analizar si los cuidadores tienen a alguna persona a su cargo, el 63,2\% no tiene ningún familiar a su cargo, el $21,1 \%$ tiene a más de un hijo mayor de edad, el $10,5 \%$ tiene a su padre, madre u otro familiar enfermo a su cargo y el 5,3\% tiene un hijo menor de edad.

Gráfico 1. Análisis descriptivo de las cargas familiares de los cuidadores

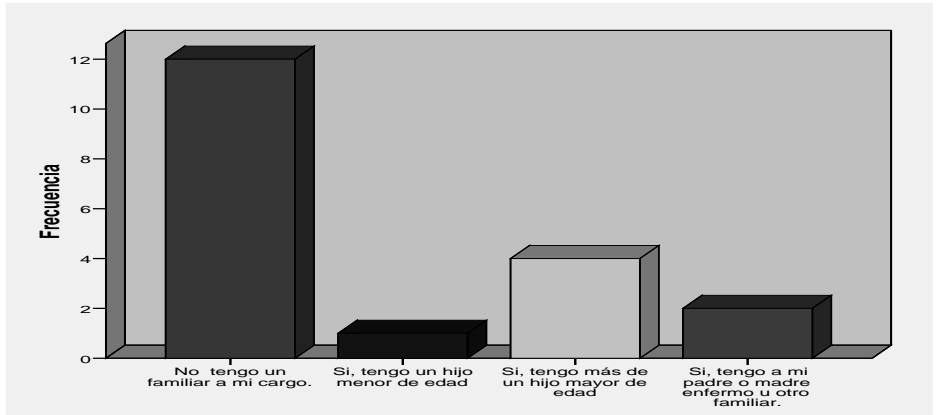

En el ámbito laboral, y con relación al tiempo trabajado con personas mayores, el 31,6\% tiene más de 10 años de experiencia, además encontramos que el $31,6 \%$ entre 5 y 10 años, el 26,3\% oscila de los 3 a los 5 años y sólo un 10,5\% tiene de 1 a 3 años de trabajo en este ámbito.

Con respecto a las faltas de asistencia en el ámbito laboral, el 15,8\% ha faltado alguna vez al trabajo en el último mes. Con respecto a las bajas laborales, el $31,6 \%$ ha solicitado una baja dos o más veces y el 36,8\% en alguna ocasión. Centrándonos en el último año, el 5,3\% la ha solicitado en al menos dos ocasiones. Más de la mitad de los partes de baja $(57,9 \%)$ se deben a una enfermedad médica y/o alguna intervención quirúrgica. Si estudiamos la percepción de peligrosidad que tiene el puesto de trabajo, el 15,8\% expresa que en ocasiones pone en riesgo su salud. Con relación a la información que el trabajador tiene sobre demencias y Alzheimer para poder realizar con éxito sus funciones, la mayoría opina que tiene suficiente aunque existen ciertos sujetos que consideran la información insuficiente (Gráfico 2).

Gráfico 2. Información del cuidador acerca de demencias y Alzheimer

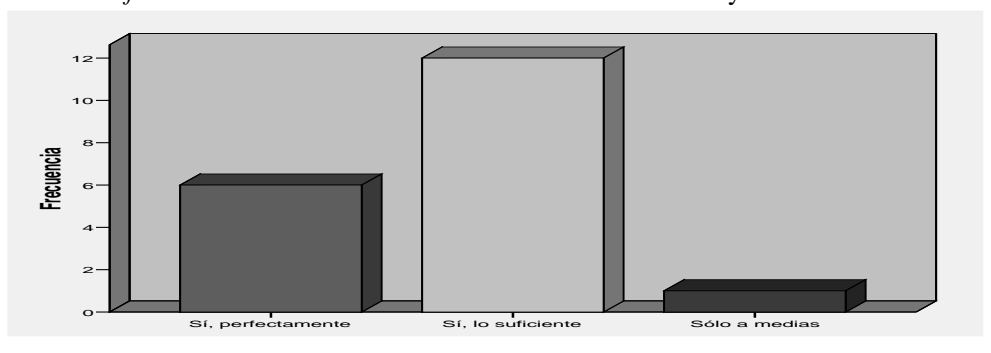


Si analizamos los hábitos de salud y consumo de sustancias en los cuidadores profesionales de la muestra, el 26,3\% fuma cigarrillos diariamente, 94,7\% expresa no beber alcohol todas las semanas y el $15,8 \%$ ha consumido tranquilizantes o pastillas para poder conciliar el sueño. Con respecto a las actividades físicas que los cuidadores profesionales realizan fuera del trabajo, el 68,4\% practica algún deporte de 1 a 3 veces por semana siendo el lugar más habitual la calle $(47,4 \%)$. El 36,8\% alude la falta de tiempo como principal motivo por el que no realiza ninguna actividad física ajena al trabajo. Con relación a las relaciones sociales, los conflictos familiares, con amigos o con el jefe obtienen el mismo porcentaje de aparición (10,5\%).

Una vez tratadas las principales variables sociodemográficas, pasamos a desarrollar el síndrome de burnout. En la Tabla 2, aparecen los principales estadísticos descriptivos para las tres dimensiones.

Tabla 2. Análisis descriptivo de las tres dimensiones del MBI en la muestra global

\begin{tabular}{|c|c|c|c|}
\hline & $\mathrm{AE}$ & $\mathrm{DP}$ & $\mathrm{RP}$ \\
\hline $\mathrm{N}$ & 19 & 19 & 19 \\
\hline Media & 10,79 & 7,63 & 36,79 \\
\hline Moda & 7 & 6 & 42 \\
\hline Desv. típ. & 6,425 & 3,578 & 4,779 \\
\hline Varianza & 41,287 & 12,801 & 22,842 \\
\hline Suma & 205 & 145 & 699 \\
\hline
\end{tabular}

Al recodificar las puntuaciones en población normal se obtienen tres niveles (bajo, medio y alto para cada dimensión). Así, los resultados muestran que la media ha sido de $1,21(D T=0,419)$ para AE, $2,11(D T=0,567)$ en DP y 2,05 $(D T=0,911)$ en RP.

Tabla 3. Porcentajes de cuidadores por nivel en cada dimensión recodificada del MBI y su comparativa por procedencia

\begin{tabular}{|c|c|c|c|c|}
\hline \multicolumn{2}{|c|}{ Niveles por dimensión } & \multirow{2}{*}{$\begin{array}{r}\text { España } \\
78.9 \%\end{array}$} & \multirow{2}{*}{$\begin{array}{c}\text { Barcelona } \\
70.0 \%\end{array}$} & \multirow{2}{*}{$\begin{array}{l}\text { Murcia } \\
88.9 \%\end{array}$} \\
\hline & Bajo & & & \\
\hline Agotamiento emocional & Medio & $21,1 \%$ & $30,0 \%$ & $11,1 \%$ \\
\hline & Alto & $0 \%$ & $0 \%$ & $0 \%$ \\
\hline \multirow{3}{*}{$\begin{array}{l}\text { Despersonalización } \\
\text { (DP) }\end{array}$} & Bajo & $10,5 \%$ & $10,0 \%$ & $11,1 \%$ \\
\hline & Medio & $68,4 \%$ & $50,0 \%$ & $88,9 \%$ \\
\hline & Alto & $21,1 \%$ & $40,0 \%$ & $0 \%$ \\
\hline \multirow{3}{*}{$\begin{array}{c}\text { Realización personal en el } \\
\text { trabajo } \\
(\mathrm{RP})\end{array}$} & Bajo & $36,8 \%$ & $60,0 \%$ & $11,1 \%$ \\
\hline & Medio & $21,1 \%$ & $30,0 \%$ & $11,1 \%$ \\
\hline & Alto & $42,1 \%$ & $10,0 \%$ & $77,8 \%$ \\
\hline
\end{tabular}

En la Tabla 3 se muestra la relación existente entre la muestra de Barcelona y la de Murcia así como de ambas con la población española. Tal como se puede observar, 
existen niveles relativamente similares en las muestras de Barcelona y Murcia, a excepción de la variable RP, en la cual, la segunda obtiene puntuaciones mayores.

Con respecto al análisis inferencial, la prueba Mann-Whitney para dos muestras independientes corrobora diferencias significativas entre la muestra murciana y catalana siendo el rango promedio en RP más alto en la primera muestra $(p=0,004)$. Usando otra prueba estadística, también volvemos a encontrar diferencias significativas entre las puntuaciones directas de ambos centros de trabajo en función de la variable "realización personal" o RP $\left(\chi^{2}{ }_{(8)}=19,000 ; p=0,015\right)$. También se aprecian dichas diferencias al analizar la RP recodificada con población española $\left(\chi_{(8)}^{2}=9,044\right.$; $\mathrm{p}=0,011$ ). No se aprecian diferencias significativas en ninguna de las tres dimensiones en función del género. También se encuentran diferencias significativas entre los niveles de $\mathrm{AE}$ y la información que tiene el trabajador en cuanto a demencias y Alzheimer $\left(\chi^{2}(1)=3,958 ; p=0,047\right)$.

El estudio pretest-postest de las puntuaciones del MBI en la muestra global refiere significatividad en la tabla de contingencia entre las puntuaciones recodificadas en la variable "despersonalización" de ambos estudios $\left(\chi^{2}{ }_{(4)}=11,720 ; p=0,020\right)$. El análisis de las variables a través del test de Wilcoxon para dos muestras relacionadas con un tamaño muestral inferior a 30, revela que existen diferencias entre las puntuaciones en agotamiento emocional de los sujetos murcianos entre el pretest y el postest ( $Z$ para la T de Wilcoxon $=-1,838 ; N=9 ; p=0,033)$. El estudio de la despersonalización a través de este test, lleva a diferencias significativas en ambas muestras, existiendo una disminución tanto en los niveles de los trabajadores de Barcelona ( $Z$ para la $\mathrm{T}$ de Wilcoxon $=-1,829 ; N=10 ; p=0,034)$ como en los de Murcia $(\mathrm{Z}$ para la $\mathrm{T}$ de Wilcoxon $=-1,992 ; N=7 ; p=0,023$ ). En cuanto a la "realización personal", los niveles en la muestra de la residencia de Barcelona presentan diferencias significativas entre el estudio piloto y el presente año, siendo más altas en la primera medida ( $\mathrm{Z}$ para la $\mathrm{T}$ de Wilcoxon $=-2,527 ; N=8 ; p=0,006)$.

Con relación al síndrome de burnout y su relación con el Trastorno por Estrés Postraumático, no se ha encontrado una relación significativa entre las dimensiones recodificadas del MBI y las puntuaciones en las escalas de "depresión”, "somatización”, "ansiedad" y "sensaciones interpersonales", ni tampoco con las puntuaciones directas de dichas dimensiones. Por otro lado, con relación al otro instrumento de evaluación usado en la investigación, el índice de correlación de Spearman refleja una relación positiva entre la "despersonalización" y la "reexperimentación" $\left(r_{s}=0,718 ; p=0,029\right)$ y la "activación" ( $\left.r_{s}=0,677 ; p=0,045\right)$. La "activación", a su vez, correlaciona con las "sensaciones interpersonales" $\left(r_{s}=0,824 ; p=0,006\right)$ y la "reexperimentación" $\left(r_{s}=0,844 ; p=0,004\right)$. 


\section{DISCUSIÓN}

El primer objetivo de nuestra investigación ha sido el estudio de los niveles actuales en las tres dimensiones de burnout en la muestra murciana y barcelonesa para estudiar su evolución. Con respecto a dicho síndrome, los cuidadores profesionales presentan diferencias significativas en función de la dimensión "realización personal en el trabajo", de forma que existe una mayor realización personal en los trabajadores de Murcia en comparación con los de la población catalana a pesar de que los primeros sufrieron una situación traumática. Este hecho puede ser explicado por la situación de crisis económica que repercute en cambios organizativos. Aquellos sujetos con puntuaciones más altas en despersonalización en el 2011, han obtenido puntuaciones más altas en el año 2012. Dicha significación se observa a través de una alta correlación entre las puntuaciones de los cuidadores profesionales de Barcelona. En esta línea, Jiménez y Cubillos (2010) reflejan en su estudio que si bien los trabajadores de su investigación presentaban un alto nivel de estrés, su satisfacción laboral no pareció verse afectada después del evento catastrófico de tanta envergadura como fue el terremoto de Chile en febrero del 2010. Los niveles en despersonalización han sido más bajos tanto en la muestra murciana como en la catalana en nuestra última investigación. Con estos datos, hemos estudiado nuestro objetivo 2, es decir, evaluar si en la población de Lorca (Murcia), los niveles hallados se han podido ver afectados por el terremoto del 11 de mayo. En la muestra de Lorca se ha encontrado que existe una relación entre la reexperimentación del suceso traumático con la despersonalización y entre ésta y un exceso de la activación.

Aquellos sujetos que puntuaron más alto en despersonalización, también lo hicieron en reexperimentación y activación, por lo que los cuidadores que tratan de forma despersonalizada y alejada al paciente como estrategia de afrontamiento frente al estrés, también tenderán a reexperimentar la situación traumática y a activarse en mayor medida que el resto.

Otro dato relevante respecto al burnout y los cuidadores, es el hecho de los cuidadores con un agotamiento emocional bajo consideran que poseen suficiente información sobre demencias y Alzheimer. En relación con este aspecto, es de destacar el valor de la formación. La formación del cuidador es un elemento primordial en el ámbito que nos atañe. Ésta, es valorada de forma positiva por los cuidadores. Así, es sabido que la formación va asociada a una mejora en la calidad laboral del cuidador (Gázquez, Pérez-Fuentes, Fernández, González, Ruiz y Díaz, 2009).

Los síntomas estudiados en relación con el TEP en la muestra de Murcia, revela que ésta está caracterizada por elevadas puntuaciones en variables como la reexperimentación, evitación e hiperactivación que se asocian a la realización personal en el trabajo. Aunque nuestro objeto de estudio no ha sido medir la prevalencia del TEP 
con todos sus criterios según el DSM-IV-TR en la muestra sino estudiar sus aspectos fundamentales, es de suponer que dicha muestra presente tasas superiores a la población normal. De hecho, la prevalencia del TEP en la población española es del 1,95\% siendo más alta en la mujer $(2,79 \%)$ en relación con el varón (1,06\%) (Haro et al., 2006).

Con respecto al potencial de aplicación posterior de los resultados de nuestro estudio y en la línea de las ideas expuestas en una revisión anterior (Ortega y López, 2004) proponemos la planificación de un horario flexible al cuidador profesional para que pueda compaginar su vida personal con la laboral, el fomento de la participación de los empleados en la toma de decisiones de aquellos aspectos del centro de trabajo que repercuta en su calidad de vida, dotación de mayor retroalimentación de su ejecución, desarrollo de un equipo de trabajo, fijación de unos límites de usuarios por cuidador para evitar la saturación de éste así como la creación de unos objetivos a corto y medio plazo que guíen el rendimiento y las tareas del cuidador profesional.

Los datos obtenidos en esta investigación suponen un punto de referencia a la hora de comprender la situación actual del colectivo laboral de cuidadores profesionales de personas con demencias y Alzheimer así como una fuente valiosa de información a la hora de desarrollar programas preventivos y de intervención más ajustados a sus necesidades. Del mismo modo, se hace imprescindible llevar a cabo un seguimiento de los síntomas de estrés postraumático presentados en la población murciana.

\section{REFERENCIAS}

Alarcón, J., Vaz, F. y Guisado, J. (2001). Análisis del síndrome de burnout: psicopatología, estilos de afrontamiento y clima social. Revista de Psiquiatría de la Facultad de Medicina Barna, 28(6), 358-381.

Alvarado, B.C., Sandín, B., Valdez-Medina, J.L., González-Arratia, N. y Rivera, S. (2012). Análisis factorial confirmatorio del Cuestionario SA-45 en una muestra mexicana. Anales de Psicología, 28(2), 426-433.

Bowman, M.L. y Yehuda, R. (2004). Risk factors and adversity-stress model. En G.M. Rosen (Ed.) Posttraumatic stress disorder. Issues and controversies. England: John Wiley \& Sons, Ltd.

Echeburúa, E., Corral, P., Amor, P.J., Zubizarreta, I. y Sarasua, B. (1997). Escala de gravedad de síntomas trastorno por estrés postraumático: propiedades psicométricas. Análisis y Modificación de Conducta, 23(90), 503-526.

Freudenberger, H. (1974). Staff burn-out. Journal of Social Issues, 30(1), 159-165.

Gázquez, J.J., Pérez-Fuentes, M.C., Lucas, F. y Yuste, N. (2008). Prevalencia de los trastornos mentales en la población mayor. Anales de Psicología, 24(2), 327-333.

Gázquez, J.J., Pérez-Fuentes, M.C., Férnandez, M., González, L., Ruiz, I. y Díaz, A. (2009). Oldage stereotypes to the gerontology education: an intergenerational study. European Journal of Education and Psychology, 2(3), 263-273. 
Guilló, P. (2002). Comprender el Alzheimer. Profesionales. Valencia: Dirección General para la Salud Pública. Servicio de Promoción de la Salud.

Haro, J.M., Palacín, C., Vilagut, G., Martínez, M., Bernal, M., Luque, I., Codony, M., Dolz, M. y Alonso, J. (2006). Prevalencia de los trastornos mentales y factores asociados: resultados del estudio ESEMeD-España. Medicina Clínica, 126, 445-51.

Jackson, S.E., Shwab, R.L. y Schuler, R.S. (1986). Toward an understanding of the burnout phenomenon. Journal of Applied Psychology, 71, 630-640.

Jiménez, A.E. y Cubillos, R.A. (2010). Estrés percibido y satisfacción laboral después del terremoto ocurrido el 27 de febrero de 2010 en la Zona Centro-Sur de Chile. Terapia psicológica, 28(2), 187-192.

Martínez, J.P. (2011). El estrés de los profesores. Un análisis sobre los factores de estrés en los profesores de secundaria de Murcia. Tesis doctoral. Universidad de Murcia.

Maslach, C. y Jackson, S.E. (1986). Maslach Burnout Inventory (2 ${ }^{\mathrm{a}}$ ed.). Palo Alto, CA: Consulting Psychologists Press.

Méndez, I., Secanilla, E., Martínez, J.P. y Navarro, J. (2011). Encuesta a trabajadores de residencias de personas mayores. No publicado.

Méndez, I., Secanilla, E., Martínez, J.P. y Navarro, J. (2011). Estudio comparativo de burnout en cuidadores profesionales de personas mayores institucionalizadas con demencias y otras enfermedades. European Journal of Investigation in Health, Psychology and Education, $1(2), 61-70$.

Olivares, V.E., Vera, A. y Juárez, A. (2009). Prevalencia del síndrome de quemarse por el trabajo (burnout) en una muestra de profesionales que trabajan con personas con discapacidad en Chile. Ciencia y Trabajo, 32, 63-71.

Ortega, C. y López, F. (2004). El burnout o síndrome de estar quemado en los profesionales sanitarios: revisión y perspectivas. International Journal of Clinical and Health Psychology, 4(1), 137-160.

Pérez, J. (2010). El envejecimiento de la población española. Investigación y Ciencia, 410, 34-42.

Sandín, B., Valiente, R.M., Chorot, P., Santed, M.A. y Lostao, L. (2008). SA-45: forma abreviada del SCL-90. Psicothema, 20(2), 290-296.

Seisdedos, N. (1997). MBI. Inventario "Burnout” de Maslach: Manual. Madrid: TEA.

Recibido: 29 de febrero de 2012 Recepción Modificaciones: 23 de marzo de 2012

Aceptado: 29 de marzo de 2012 